\title{
TIME REVERSAL BEAMFORMING FOR MICROWAVE BREAST CANCER DETECTION
}

\author{
Yuanwei Jin, Yi Jiang, and José M.F. Moura \\ Carnegie Mellon University \\ Department of Electrical and Computer Engineering \\ Pittsburgh, PA 15213
}

\begin{abstract}
Microwave radiation is well known as a diagnostic imaging method for many medical applications, for example, early stage breast cancer detection. Microwave detection of breast tumors is a non-ionising, potentially low cost, in vivo modality that relies on the dielectric contrast between healthy and malignant breast tissues. The scattering environment in a breast often appears to be inhomogeneous due to changing dielectric properties of the breast tissues. Time reversal (TR) is an adaptive waveform transmission scheme that utilizes the rich scattering medium to best match to the target response. In this paper, we develop the microwave time reversal beamformer for breast tumor detection. The TR beamforming scheme is examined based upon a breast model using the twodimensional finite-difference time-domain (FDTD) method. We show that time reversal microwave beamforming is a more robust, higher resolution imaging scheme than conventional beamforming schemes.
\end{abstract}

Index Terms - Biomedical imaging, Microwave imaging

\section{INTRODUCTION}

X-ray mammography is the most common method to detect early stage breast cancer. Although mammography provides high quality images at low radiation doses in the majority of patients, it has limitations. Between $10-30 \%$ of women with breast cancer who undergo mammography have negative mammograms. On the other hand, approximately two-thirds of all breast lesions that are identified by mammography and biopsied turn out to be benign. The goal of increasing diagnostic accuracy and reducing patient morbidity motivates the search for alternative technologies for early breast cancer detection. One such alternative is microwave imaging.

It has been long recognized that there is the potential of microwave radiation for diagnostic imaging [1], for example, tomography [2], ultra-wideband radar technique [3], and beamforming [4]. The microwave exam involes the propagation of very low levels of microwave energy through breast tissue to measure electrical properties. The physical basis for microwave imaging is the significant contrast of the dielectric properties between malignant and normal breast tissues [5] Generally, tumors have been found to have more water and blood than regular tissue. Among the proposed microwave

This work is funded by the Defence Advanced Research Projects Agency through the Army Research Office under grant no. W911NF-04-1-0031. imaging techniques, the beamforming approach seeks to identify the presence and location of significant backscattered energy from malignant breast tumors using controlled microwave sources. For a beamforming imager, each antenna transmits a low power ultrawide band signal into the breast and records the backscatter. The backscatter signals are passed through the beamformer, followed by a detector. By scanning through the whole imaged area, the output of the detector produces an image of the backscattered energy as a function of location in the breast. Having a significant dielectric contrast with normal breast tissues, malignant tumors produce localized regions of relatively large backscatter energy and stand out in the image.

Breast tissues are generally inhomogeneous. The skin layer and the small variation of the dielectric properties of the breast tissues are the main source of clutter, which degrades the formed breast images. Time reversal is an adaptive waveform transmission scheme that best matches to the target response. An electromagnetic TR experiment was reported in [6]. We have developed time reversal beamforming technique and showed that it successfully mitigates clutter and focus on target [7]. The resulting images show higher resolution than conventional beamformers. To study the feasibility of time reversal beamforming for locating breast tumors, we use the FDTD, a computational electrodynamics modeling technique, to simulate the interactions between electromagnetic waves and the surrounding breast tissues.

\section{TIME REVERSAL BEAMFORMING}

We developed time reversal beamforming for locating targets using a multi-static configuration in [7]. We use array $\mathrm{A}$ with antennas $A_{1}, \cdots, A_{N}$, and array $\mathrm{B}$ with antennas $B_{1}, \cdots, B_{N}$ placed at different locations. Both can switch between transmit mode and receive mode. The probing signal is $s(t), t \in$ $\left[0, T_{0}\right]$. Its Fourier transform is $S(\omega), \omega \in\left[\omega_{0}, \omega_{0}+B\right]$. The signal has duration $T_{0}$ and bandwidth $B$. Time reversal of a real-valued signal becomes phase conjugation in the frequency domain, i.e., $s(-t)$ is equivalent to $S^{*}(\omega)$. Written in a discrete form, the probing signal becomes

$$
S\left(\omega_{q}\right), 0 \leq q \leq Q-1, \omega_{q}=\frac{2 \pi}{T_{0}(Q-1)}\left(q_{1}+q\right),
$$

where $q_{1}$ is a constant. In what follows, we will use the discrete frequency representation to develop our imaging algorithms. 
Transmitting a wideband pulse from the transmitter $A_{j}$, we record the backscattered wave field reflected from scatterers in the breast at the receiver $B_{i}$. Thus, we can obtain a multi-static data matrix at frequency $\omega_{q}$ as follows:

$$
\left[\mathbf{K}_{t}\left(\omega_{q}\right)\right]_{i j}=G\left(\mathbf{r}_{B_{i}}, \mathbf{x}_{t} ; \omega_{q}\right) \tau\left(\mathbf{x}_{t} ; \omega_{q}\right) G\left(\mathbf{x}_{t}, \mathbf{r}_{A_{j}} ; \omega_{q}\right),
$$

where $G\left(\mathbf{r}, \mathbf{r}^{\prime} ; \omega_{q}\right)$ is the Green's function between transmitter $\mathbf{r}^{\prime}$ and receiver $\mathbf{r}$ at frequency $\omega_{q}$. For a reciprocal channel, the Green's function satisfies $G\left(\mathbf{r}, \mathbf{r}^{\prime} ; \omega_{q}\right)=G\left(\mathbf{r}^{\prime}, \mathbf{r} ; \omega_{q}\right)$. An example of Green's function of a medium with relative permittivity $\epsilon_{r}$ and relative permeability $\mu_{r}$ is

$$
G\left(\mathbf{r}, \mathbf{r}^{\prime} ; \omega_{q}\right)=\frac{1}{4 j} H_{0}^{(2)}\left(k_{q}\left|\mathbf{r}-\mathbf{r}^{\prime}\right|\right),
$$

where $H_{0}^{(2)}$ is the zeroth-order Hankel function of the second kind. $k_{q}=\omega_{q} / v$ is the wavenumber of a propagating wave with angular frequency $\omega_{q}$, and $v$ is the medium propagation velocity, $v=\frac{1}{\sqrt{\epsilon_{r} \mu_{r}}} c, c$ is the speed of light. In this paper, we assume that $\mu_{r}=1$, thus $v=\frac{1}{\sqrt{\epsilon_{r}}} c$. Eqn. (2) leads to a revealing subspace representation of the response matrix:

$$
\mathbf{K}_{t}\left(\omega_{q}\right)=\tau\left(\mathbf{x}_{t} ; \omega_{q}\right) \mathbf{g}_{B}\left(\mathbf{x}_{t} ; \omega_{q}\right) \mathbf{g}_{A}^{T}\left(\mathbf{x}_{t} ; \omega_{q}\right),
$$

where the Green's function vectors are given by

$$
\begin{aligned}
& \mathbf{g}_{B}\left(\mathbf{x}_{t} ; \omega_{q}\right)=\left[G\left(\mathbf{r}_{B_{0}}, \mathbf{x}_{t} ; \omega_{q}\right), \cdots, G\left(\mathbf{r}_{B_{N-1}}, \mathbf{x}_{t} ; \omega_{q}\right)\right]^{T} \\
& \mathbf{g}_{A}\left(\mathbf{x}_{t} ; \omega_{q}\right)=\left[G\left(\mathbf{x}_{t}, \mathbf{r}_{A_{0}} ; \omega_{q}\right), \cdots, G\left(\mathbf{x}_{t}, \mathbf{r}_{A_{P-1}} ; \omega_{q}\right)\right]^{T}
\end{aligned}
$$

Time reversal iteratively probes the scattering environment to suppress clutter response and to focus on intended targets. To distinguish the clutter response and the target response, we adopt two scattering wave-field representations, $\mathbf{K}_{c}\left(\omega_{q}\right)$ (i.e., clutter only response), and $\mathbf{K}_{c+t}\left(\omega_{q}\right)$ (i.e., clutter plus target response). The effective target response can be written as

$$
\mathbf{K}_{t}\left(\omega_{q}\right)=\mathbf{K}_{t+c}\left(\omega_{q}\right)-\mathbf{K}_{c}\left(\omega_{q}\right) .
$$

In a rich scattering environment, $\mathbf{K}_{t}\left(\omega_{q}\right)$ includes both the direct reflection from the target and the secondary reflection due to surrounding clutter.

The proposed time reversal scheme consists of the following five steps:

Step-1: Clutter probing This is the training step and we assume that only the clutter exists. This step provides an initial estimate of the clutter response matrix termed as $\mathbf{K}_{c}\left(\omega_{q}\right)$. In reality, the clutter response will be estimated. We will address this issue in section 4

Step-2: Time reversal clutter suppression In this step, we use an adaptive filter to suppress the clutter return. The filter is designed by minimizing the total clutter returns. This is done by, in contrast to focusing in time reversal, anti-focusing the clutter through an adaptive filter [7]

$$
\mathbf{W}\left(\omega_{q}\right)=k_{q}\left[\mathbf{K}_{c}^{*}\left(\omega_{q}\right) \mathbf{K}_{c}^{T}\left(\omega_{q}\right)\right]^{\dagger},
$$

where $\dagger$ is the pseudo-inverse, $k_{q}$ is a scaling factor. In a rich scattering environment where $\mathbf{K}_{c}\left(\omega_{q}\right)$ is full rank, the filter (6) becomes a whitening filter, hence the clutter return is whitened and then subtracted out in succeeding processing steps.

Step-3: Target monitoring In this step, we continue probe the environment in which the target is present or absent using signal (1). We apply the filter (6) to the received backscatter signals, suppress, and then subtract out the clutter.

Step-4: Time reversal target focusing After the whitened clutter is subtracted out, the residual target signal is time reversed (or phase conjugated in frequency domain). Finally, we obtain the clutter suppressed and target focused data matrices measured at array A and array B, respectively.

$$
\begin{aligned}
& \mathbf{M}^{B}\left(\omega_{q}\right)=k_{q} \mathbf{K}_{t}\left(\omega_{q}\right) \mathbf{K}_{t}^{H}\left(\omega_{q}\right) \mathbf{K}_{c}^{-H}\left(\omega_{q}\right) S\left(\omega_{q}\right)(7) \\
& \mathbf{M}^{A}\left(\omega_{q}\right)=k_{q} \mathbf{K}_{t}^{T}\left(\omega_{q}\right) \mathbf{K}_{t}^{*}\left(\omega_{q}\right) \mathbf{K}_{c}^{-*}\left(\omega_{q}\right) S\left(\omega_{q}\right)
\end{aligned}
$$

Step 5: Image formation. This final step forms the image by scanning the area of interest with two focused beams, one at array $B$ and the other at array $A$. The beam at $B$ is when we start at $B$ and end at $B$; similarly, the beam at $A$ is when we start at $A$ and end at $A$. Next, we calculate beamformer weight vectors to extract target locations. Let $\mathbf{w}_{r B}\left(\mathbf{x} ; \omega_{q}\right)$, $\mathbf{w}_{t B}\left(\mathbf{x} ; \omega_{q}\right), \mathbf{w}_{r A}\left(\mathbf{x} ; \omega_{q}\right)$, and $\mathbf{w}_{t A}\left(\mathbf{x} ; \omega_{q}\right)$ denote the receive and transmit beams for arrays $B$ and $A$, respectively, at frequency $\omega_{q}, \forall q$.

$$
\begin{aligned}
\mathbf{w}_{r B}\left(\mathbf{x} ; \omega_{q}\right) & =\frac{\mathbf{g}_{B}\left(\mathbf{x} ; \omega_{q}\right)}{\left\|\mathbf{g}_{B}\left(\mathbf{x} ; \omega_{q}\right)\right\|}, \\
\mathbf{w}_{t B}\left(\mathbf{x} ; \omega_{q}\right) & =\frac{\mathbf{K}_{c}^{-1}\left(\omega_{q}\right) \mathbf{g}_{B}\left(\mathbf{x} ; \omega_{q}\right)}{\left\|\mathbf{K}_{c}^{-1}\left(\omega_{q}\right) \mathbf{g}_{B}\left(\mathbf{x} ; \omega_{q}\right)\right\|}, \\
\mathbf{w}_{r A}\left(\mathbf{x} ; \omega_{q}\right) & =\frac{\mathbf{g}_{A}\left(\mathbf{x} ; \omega_{q}\right)}{\left\|\mathbf{g}_{A}\left(\mathbf{x} ; \omega_{q}\right)\right\|}, \\
\mathbf{w}_{t A}\left(\mathbf{x} ; \omega_{q}\right) & =\frac{\mathbf{K}_{c}^{-T}\left(\omega_{q}\right) \mathbf{g}_{A}\left(\mathbf{x} ; \omega_{q}\right)}{\left\|\mathbf{K}_{c}^{-T}\left(\omega_{q}\right) \mathbf{g}_{A}\left(\mathbf{x} ; \omega_{q}\right)\right\|}
\end{aligned}
$$

Applying the weight vectors to data matrices $\mathbf{M}^{B}\left(\omega_{q}\right)$ and $\mathbf{M}^{A}\left(\omega_{q}\right)$, we obtain the complex output of the beamformers $B$ and $A$

$$
\begin{aligned}
& Y^{B}\left(\mathbf{x} ; \omega_{q}\right)=\mathbf{w}_{r B}^{H}\left(\mathbf{x} ; \omega_{q}\right) \mathbf{M}^{B}\left(\omega_{q}\right) \mathbf{w}_{t B}\left(\mathbf{x} ; \omega_{q}\right)( \\
& Y^{A}\left(\mathbf{x} ; \omega_{q}\right)=\mathbf{w}_{r A}^{H}\left(\mathbf{x} ; \omega_{q}\right) \mathbf{M}^{A}\left(\omega_{q}\right) \mathbf{w}_{t A}\left(\mathbf{x} ; \omega_{q}\right)(
\end{aligned}
$$

We now combine the outputs of these two beamformers by triangulation, i.e., we multiply the outputs of the two beamformers at each frequency to form the final image $I(\mathbf{x})$ as a function of each pixel $\mathrm{x}$ by implementing the energy detector

$$
I(\mathbf{x})_{\mathrm{TRBF}}=\sum_{q=0}^{Q-1}\left|Y^{A}\left(\mathbf{x} ; \omega_{q}\right) Y^{B}\left(\mathbf{x} ; \omega_{q}\right)\right|^{2}
$$

\section{FDTD SIMULATION MODELS}

Next, we discuss the FDTD simulation modeling. 


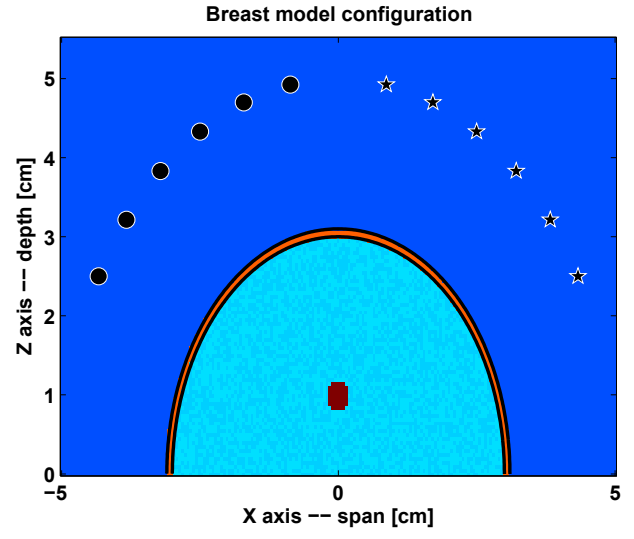

Fig. 1. Simplified breast model. Two antenna arrays, A and $\mathrm{B}$, denoted by the circles and the pentagrams, respectively, are placed on the surface of the breast. The thick layer of $1 \mathrm{~mm}$ is the skin. Normal breast tissues are inside the skin layer. A tumor is surrounded by normal tissues.

\subsection{System configuration}

We use a simplified breast model to carry out FDTD simulations (see Fig. 1). A patient lies in a prone position with the breasts immersed in a liquid. The liquid has similar electrical properties to the breast tissue, but has lower loss. Two arrays of six antennas, A and B, are placed in the liquid, and positioned in a circle around the breast. The antennas are $2 \mathrm{~cm}$ away from the breast surface. The data is acquired in a multistatic fashion. Signals are transmitted from array A, one antenna at a time, and recorded by all the antennas of array B.

\subsection{Breast model}

A breast may include normal breast tissue, malignant tumor, and skin. The breast model shown in Fig. 1 is a half sphere of a diameter $6 \mathrm{~cm}$. Although this model is not realistically shaped, it is a reasonable simplification for malignant tumor detection in two-dimensional cross sections.

Next, we choose the electrical properties of the breast model. Breast tissue dielectric properties, including the relative dielectric permittivity, $\epsilon_{r}$, and conductivity $\sigma$, at various frequency ranges are measured and studied by many researchers (see, e.g. [5]). In this paper, we choose the breast tissue dielectric data similar to those selected by [3]. For simplicity, tissue dielectric properties are assumed to be frequency independent. The electrical properties of the normal breast tissues are $\epsilon_{r}=9, \sigma=0.4 \mathrm{~S} / \mathrm{m}$. We introduce a $16 \%$ upper bound of variability in the breast tissue to represent the inhomogeneities. The skin is modeled as a 1-mm thick layer with $\epsilon_{r}=36, \sigma=4 \mathrm{~S} / \mathrm{m}$. The tumor is modeled as a sphere of diameter 2-mm with $\epsilon_{r}=50, \sigma=4 \mathrm{~S} / \mathrm{m}$. The antenna arrays are placed in a liquid with $\epsilon_{r}=9, \sigma=0 \mathrm{~S} / \mathrm{m}$. We will examine two cases for simulation. One is when the conductivity of all the tissues are zero, which represents a reciprocal and lossless channel. The other is when the conductivity is non-zero and indicates a weakly lossy channel.

\subsection{FDTD Modeling}

We developed a two-dimensional FDTD model of the breast in Fig. 1 to simulate the back-propagated wave field. The FDTD grid cell size is chosen $0.5 \times 0.5 \mathrm{~mm}^{2}$. The resulting FDTD grid is terminated with the Perfect Matched Layer (PML) absorbing boundary condition. The probing signal is a sinc signal of $2 \mathrm{GHz}$ bandwidth with center frequency of 9 GHz. The FDTD simulated data are time domain samples, and can be converted to frequency domain via the Fourier transform.

\section{PERFORMANCE RESULTS}

We now test our beamforming algorithm using simulated data by the FDTD method. In an ideal case, we can extract the tumor signature response by subtracting the wave fields calculated from a model with and without the tumor, i.e., target response $\mathbf{K}_{t}\left(\omega_{q}\right)$ defined in (5). We implement the TR beamformer and compare it to the conventional beamformer defined as follows:

$$
I(\mathbf{x})_{\mathrm{DSBF}}=\sum_{q=0}^{Q-1}\left|\mathbf{g}_{A}\left(\mathbf{x} ; \omega_{q}\right) \mathbf{K}_{t}\left(\omega_{q}\right) \mathbf{g}_{B}\left(\mathbf{x} ; \omega_{q}\right)\right|^{2} .
$$

In practice, we must estimate the clutter response. The main source of the clutter is the skin layer and the random variation of the dielectric properties of the breast tissue. From our simulated FDTD time domain waveform, we observe that the returned signal has a strong leading reflection followed by other reflections. This strong reflection is caused by the skinbreast interface, while the trailing reflections are due to either the tumor or the breast tissues. Thus, we can apply a time domain gating scheme to isolate the strong skin reflections, i.e., the dominant clutter, and estimate it across the receive array. The estimated clutter response is used to construct the clutter whitening filter. We show that this adaptive time reversal clutter mitigation scheme results in a better performance compared with a direct subtraction scheme.

The layered structure of the breast model imposes difficulty in computing the Green's function. In our model, the dielectric properties of the liquid and the average breast tissue is the same, but the skin is different. Thus, the skin manifests itself as an inhomogeneous medium. However, the thickness of the skin is small relative to the wavelength. Thus, the overall influence of the skin on the Green's function can be approximated as a decaying factor.

Fig. 2 shows the images produced by the TR beamformer and the conventional beamformer when the conductivity constants are set to zero. Thus, the scattering medium is lossless and the reciprocity holds. The image produced by the TR beamformer has a dynamic range of $50 \mathrm{~dB}$, while the conventional image is of $32 \mathrm{~dB}$. For comparison purposes, both images are properly scaled to the same range. The sharper, higher contrast image has more details and thus a better resolution. For all the the images, the cross indicates the exact tumor location, while the circle is the peak of the image. Fig. 2 shows that the TR beamformer image has a better accuracy 

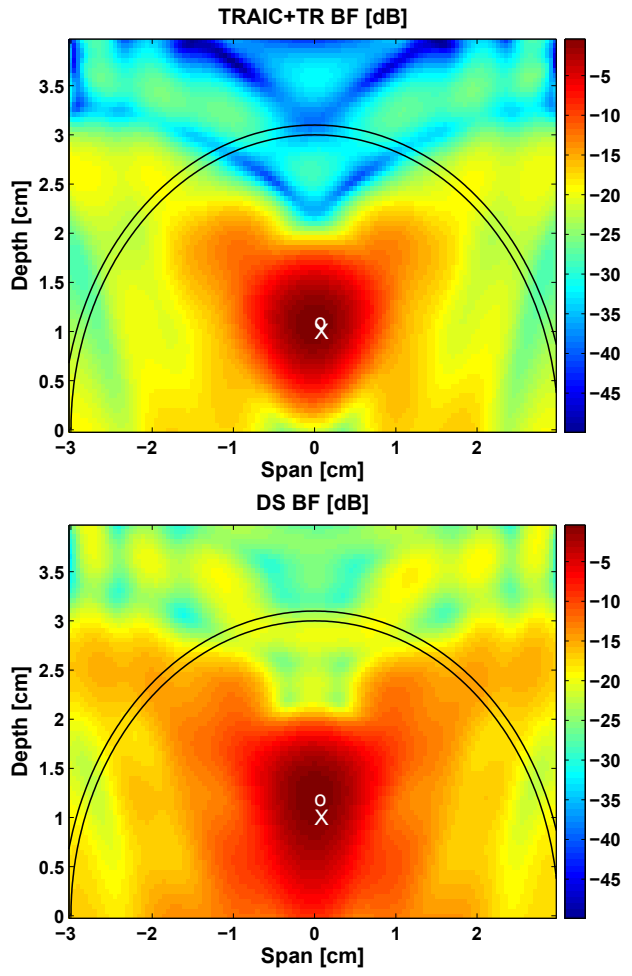

Fig. 2. Upper-TR beamforming; Lower-Conventional beamforming. Conductivity is zero.
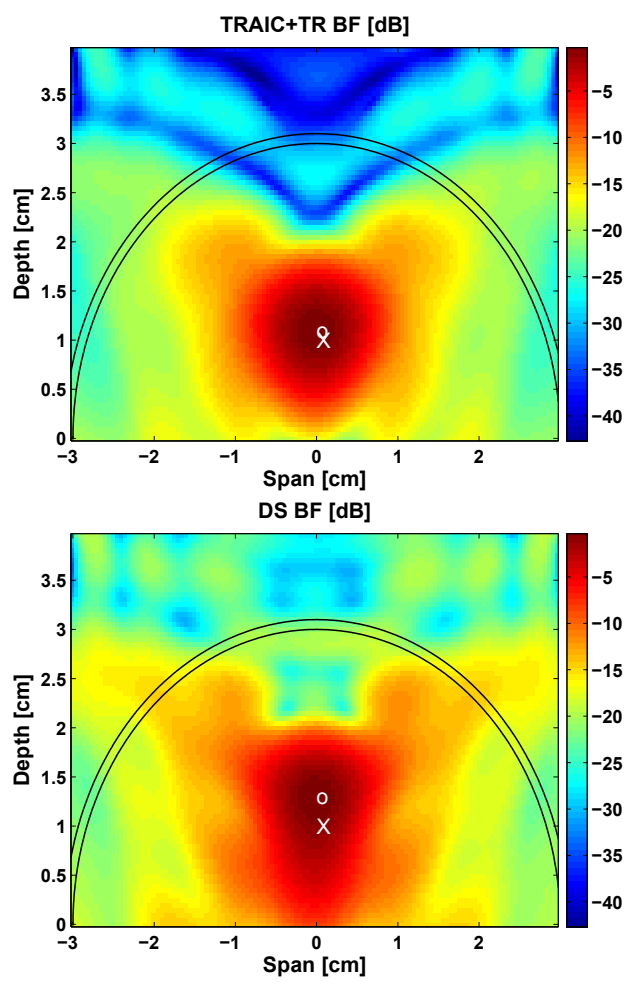

Fig. 3. Upper-TR beamforming; Lower-Conventional beamforming. Conductivity is non-zero.
(1 $\mathrm{mm}$ bias) than the conventional beamformer image $(2 \mathrm{~mm}$ bias).

Fig. 3 shows the images produced by the TR beamformer and the conventional beamformer when the breast tissues have non-zero conductivity. This represents a relatively weak conducting medium of the breast. Again, the TR beamformer image has a dynamic range of $42 \mathrm{~dB}$ compared to the conventional image of $26 \mathrm{~dB}$. The bias is $1 \mathrm{~mm}$ for the TR beamforming image, and $3 \mathrm{~mm}$ for the conventional beamforming image. The results also show that TR beamformer can compensate for moderately conductive media, and still demonstrates its high resolution imaging capability. In the case of a strongly conducting medium, the reversed time Cauchy problem is ill-posed and unstable.

\section{CONCLUSION}

We examine the feasibility of time reversal beamforming for early stage breast cancer detection. We choose a reasonable breast model and use the FDTD method to simulate the wave propagation in the breast tissues. The imaging results demonstrate that the proposed TR beamforming is a robust, high resolution imaging scheme for detecting early stage breast cancer. Future research is how to estimate the clutter response and adapt the proposed TR beamforming scheme in vivo.

\section{REFERENCES}

[1] L. E. Larsen and J. H. Jacobi, Medical Applications of Microwave Imaging. New York, NY: IEEE Press, 1986.

[2] P. M. Meaney, M. W. Fanning, D. Li, S. P. Poplack, and K. D. Paulsen, "A clinical prototype for active microwave imaging of the breast," IEEE Transactions on Microwave Theory and Techniques, vol. 48, pp. 1841-1853, November 2000 .

[3] E. C. Fear and M. A. Stuchly, "Microwave detection of breast cancer," IEEE Transactions on Microwave Theory and Techniques, vol. 48, no. 11, pp. 1854-1863, August 2000 .

[4] E. J. Bond, X. Li, S. C. Hagness, and B. D. V. Veen, "Microwave imaging via space-time beamforming for early detection of breast cancer,' IEEE Transactions on Antennas and Propagation, vol. 51, no. 8, pp. 1690-1705, August 2003.

[5] A. J. Surowiec, S. S. Stuchly, J. R. Barr, and A. Swarup, "Dielectric properties of breast carcinoma and the surrounding tissues," IEEE Transactions on Biomedical Engineering, vol. 35, no. 4, pp. 257-263, April 1988.

[6] B. E. Henty and D. D. Stancil, "Multipath enabled super-resolution for RF/microwave communication using phase-conjugate arrays," Physics Review Letters, vol. 93, no. 24, p. 243904, December 2004.

[7] J. M. F. Moura and Y. Jin, "Time reversal imaging by adaptive interference cancellation," IEEE Transactions on Signal Processing, 2007, submitted for publication. 